\title{
Factors associated with additional anterior cruciate ligament reconstruction and register comparison: a systematic review on the Scandinavian knee ligament registers
}

\author{
Eleonor Svantesson, ${ }^{1}$ Eric Hamrin Senorski, ${ }^{2}$ Angelo Baldari, ${ }^{3}$ Olufemi R. Ayeni, ${ }^{4}$ \\ Lars Engebretsen, ${ }^{5,6}$ Francesco Franceschi, ${ }^{3}$ Jon Karlsson, ${ }^{1,7}$ Kristian Samuelsson ${ }^{1,7}$
}

- Additional material is published online only. To view, please visit the journal online (http://dx.doi.org/10.1136/ bjsports-2017-098192).

${ }^{1}$ Department of Orthopaedics, Institute of Clinical Sciences,

The Sahlgrenska Academy, University of Gothenburg, Göteborg, Sweden

${ }^{2}$ Department of Health and Rehabilitation, Institute of Neuroscience and Physiology, The Sahlgrenska Academy, University of Gothenburg, Göteborg, Sweden

${ }^{3}$ Department of Orthopaedic and Trauma Surgery, Campus Bio-Medico University of Rome, Rome, Italy

${ }^{4}$ Division of Orthopaedic Surgery, Department of Surgery, McMaster University, Hamilton, Ontario, Canada

${ }^{5}$ Orthopedic Clinic, Oslo

University Hospital, University of Oslo, Oslo, Norway

${ }^{6}$ OSTRC, The Norwegian School of Sports Sciences, Oslo, Norway ${ }^{7}$ Department of Orthopaedics, Sahlgrenska University Hospital, Mölndal, Sweden

\section{Correspondence to}

Eleonor Svantesson, Department of Orthopedics, Institute of Clinical Sciences, Sahlgrenska Academy, University of Gothenburg, Gothenburg, SE431 80, Sweden; eleonor.svantesson@outlook. com

Accepted 21 June 2018 Published Online First 17 July 2018

Check for updates

(C) Author(s) (or their employer(s)) 2018. No commercial re-use. See rights and permissions. Published by BMJ.

To cite: Svantesson $\mathrm{E}$, Hamrin Senorski E, Baldari A, et al. Br J Sports Med 2019:53:418-425.

\begin{abstract}
Objective To present an overview of the Scandinavian knee ligament registers with regard to factors associated with additional $A C L$ reconstruction, and studies comparing the Scandinavian registers with other knee ligament registers.
\end{abstract}

Design Systematic review.

Data sources Four electronic databases: PubMed, EMBASE, the Cochrane Library and AMED were searched, and 157 studies were identified. Two reviewers independently screened titles, abstracts and full-text studies for eligibility. A modified version of the Downs and Black checklist was applied for quality appraisal.

Eligibility criteria for selecting studies Eligible studies were those published since the establishment of the Scandinavian registers in 2004, which reported factors associated with additional $A C L$ reconstruction and compared data from other registers.

Results Thirty-one studies met the inclusion criteria and generally displayed good reporting quality. Adolescent age ( $<20$ years) was the most common factor associated with additional $A C L$ reconstruction. The choice of hamstring tendon graft compared with patella tendon, transportal femoral tunnel drilling, smaller graft diameter and utilisation of suspensory fixation devices were associated with additional $A C L$ reconstruction. Concomitant cartilage injury decreased the likelihood of additional $A C L$ reconstruction. Patient sex alone did not influence the likelihood. The demographics of patients undergoing $\mathrm{ACL}$ reconstruction in the Scandinavian registers are comparable to registers in other geographical settings. However, there are differences in surgical factors including the presence of intra-articular pathology and graft choice.

Summary The studies published from the Scandinavian registers in general have a high reporting quality when regarded as cohort studies. Several factors are associated with undergoing additional $A C L$ reconstruction. The results from the registers may help facilitate treatment decisions.

\section{INTRODUCTION}

National registers of patients undergoing ACL reconstruction have been established for $>10$ years in Scandinavia. The compliance and completeness of the registers at baseline are high. $^{1-3}$ Over $90 \%$ of the annually performed ACL reconstructions in Sweden are registered in the Swedish National Knee Ligament Register (SNKLR), an almost 100\% compliance has been reported from the Norwegian Knee Ligament Register (NKLR) ${ }^{4}$ and the registration completeness in the Danish Knee Reconstruction Register (DKRR) has ranged between $85 \%$ and $92 \%$ over the past years. ${ }^{5}$ The registers aim to improve clinical outcomes by providing continued feedback to hospitals and surgeons with regard to surgical techniques and devices that may lead to premature failure, and to distinguish predictors of good and poor outcomes. ${ }^{36}$

More than 70000 primary ACL reconstructions in the Scandinavian knee ligament registers $^{7}$ enable robust investigation of epidemiology and factors associated with undergoing a subsequent additional ACL reconstruction (revision or contralateral ACL reconstruction). The DKRR also includes data on residual laxity after primary ACL reconstruction, ${ }^{8}$ which can identify treatment failures and inferior outcomes that do not proceed to revision. Increased knowledge of factors associated with additional ACL reconstruction or residual laxity entails that such factors could be targeted in the clinical care of these patients and potentially prevents a second ACL reconstruction. Ultimately, such knowledge should improve treatment in Scandinavia, and in other population settings. Therefore, understanding how patient-related and surgery-related factors in Scandinavia compare with cohorts in other geographical areas could aid in determining the generalisability of conclusions drawn from the Scandinavian knee ligament registers and the general clinical applicability of the findings.

The purpose of this study was to present an overview of findings published from the Scandinavian knee ligament registers on factors associated with undergoing additional ACL reconstruction or residual laxity, and to present studies that compare data between Scandinavian registers and other large knee ligament registers.

\section{Specific research questions}

- Which patient-related, injury-related and surgery-related factors are associated with revision and contralateral ACL reconstruction in the Scandinavian knee ligament registers? 
- How is patient-reported outcome related to undergoing additional ACL reconstruction in the Scandinavian knee ligament register?

- Are the patient cohorts and the surgical procedures within the Scandinavian knee ligament registers comparable with other large knee ligament registers?

\section{METHODS}

This systematic review was conducted according to the Preferred Reporting Items for Systematic Reviews and Meta-Analyses guidelines.

\section{Eligibility criteria}

All original studies written in English from the Danish, Norwegian or Swedish knee ligament registers with a specific question regarding ACL reconstruction were eligible. Furthermore, a study was required to present outcomes or data on any of the following topics: additional ACL reconstruction-defined as revision or contralateral ACL reconstruction following index reconstruction. Patient-reported outcome in relation to additional ACL reconstruction - the tools used for subjective outcome in the registers are the Knee Injury and Osteoarthritis Outcome Score (KOOS) and/or the European quality of life-five dimensions questionnaire and/or Tegner activity scale. Residual knee laxity following ACL reconstruction-rotatory and/or anteroposterior instability assessed by manual examination. Register comparison-defined as studies with a comparative analysis of data from any Scandinavian register to any other knee ligament register outside Scandinavia. Conference abstracts, review studies or clinical commentary articles were excluded.

\section{Information sources/literature search}

The literature search was performed by an expert in electronic search methods at the Sahlgrenska University Hospital library on 9 May 2017. An updated literature search was performed on 20 April 2018. We searched the PubMed, EMBASE, The Cochrane Library and AMED electronic databases. Search terms were mapped to relevant MeSH terms or subject headings where possible. Search terms were entered into the database under three concepts: concept 1-'Register', 'registry', 'registers' and 'registries'. Concept 2-'Sweden', 'Swedish', 'Denmark', 'Danish', 'Norway', 'Norwegian', 'Scandinavia', 'Scandinavian' and 'Nordic countries'. Concept 3-'Anterior cruciate ligament', 'Anterior cruciate ligament injuries', 'Anterior cruciate ligament reconstruction', 'Posterior cruciate ligament' and 'Posterior cruciate ligament reconstruction'. Keywords in each concept were grouped with the 'OR' operator. The results from each concept were then combined with the 'AND' operator to produce the search strategy and the final yield (see online supplementary table 1). In addition, the register holder of each Scandinavian register was contacted via email and asked to provide a list of publications from the register. Two authors (EHS and ES) independently screened all abstracts and full texts, where needed, to identify eligible articles.

\section{Data collection process}

Data were extracted independently by two authors (ES and EHS). Disagreements were resolved by discussion, or by consulting the senior author (KS). An electronic piloted form was created in Microsoft Excel for Windows (V.14.0.7, Microsoft, Redmond, Washington, USA) for data collection.

\section{Outcome}

The primary outcomes were additional ACL reconstruction (either revision or contralateral ACL reconstruction) or residual laxity. In addition, all patient-related, injury-related and surgery-related factors and outcomes reported in relation to additional ACL reconstruction were extracted and reported.

\section{Quality assessment}

There is no standardised method for assessing internal validity (bias) in register studies. Therefore, we used the Downs and Black checklist for randomised and non-randomised studies that primarily assess the reporting quality of studies. ${ }^{9}$ Two reviewers independently assessed each study. The original checklist comprises 27 items that are scored on a $0-2$ scale, yielding a maximum score of 30 points. Items related to randomisation were not applicable and items 14, 15, 23 and 24 were therefore excluded. Item 27 (power analysis) and item 21 were excluded. Item 21 was excluded since all studies aiming to analyse two or more registers would score zero (patients not recruited from the same population), even though the quality of the multiregister studies could be high. The modified checklist comprised a maximum score of 22 points.

\section{Data synthesis}

A qualitative data synthesis approach was selected because of (1) overlapping participants in multiple studies would result in including data from some participants more than once in a quantitative analysis, and (2) different methods, for example, follow-up lengths and statistical methods, have been used to address similar research questions and outcomes among the studies. Therefore, the results were summarised descriptively under the following main sections: relationship between patient-related factors and revision ACL reconstruction, relationship between surgical factors and revision ACL reconstruction, factors associated with contralateral ACL reconstruction, factors for residual laxity in primary and revision ACL reconstruction, and the Scandinavian registers compared with data from other geographical areas. Each main section was further stratified in subheadings according to each factor, for example, 'age' or 'graft type'. In cases where the studies overlapped, the result from the study with the largest cohort was considered of highest evidence in the summary, and secondarily, the study with the highest Downs and Black score was considered. A summary of extracted data is available in online supplementary table 2 .

\section{RESULTS}

The literature searches yielded in total 157 individual studies for which the first round of screening was performed. One additional study was identified via the publication lists provided by the register holders. After applying the selection criteria, a total of 31 studies were included in this systematic review (figure 1 ).

The Downs and Black score ranged from 9 to 19 , with a median score of 16 out of a possible 22 points (table 1 ). For some studies, the study design made it difficult to apply many of the items and the score as a quality indicator should therefore be interpreted with care for these studies. ${ }^{57810}$ Items 8 (adverse events reported) and 19 (compliance reliability reported) were not fulfilled by any study. With the exception of these, ensuring the representativeness of the recruited subjects (item 12) and accounting for losses to follow-up (item 


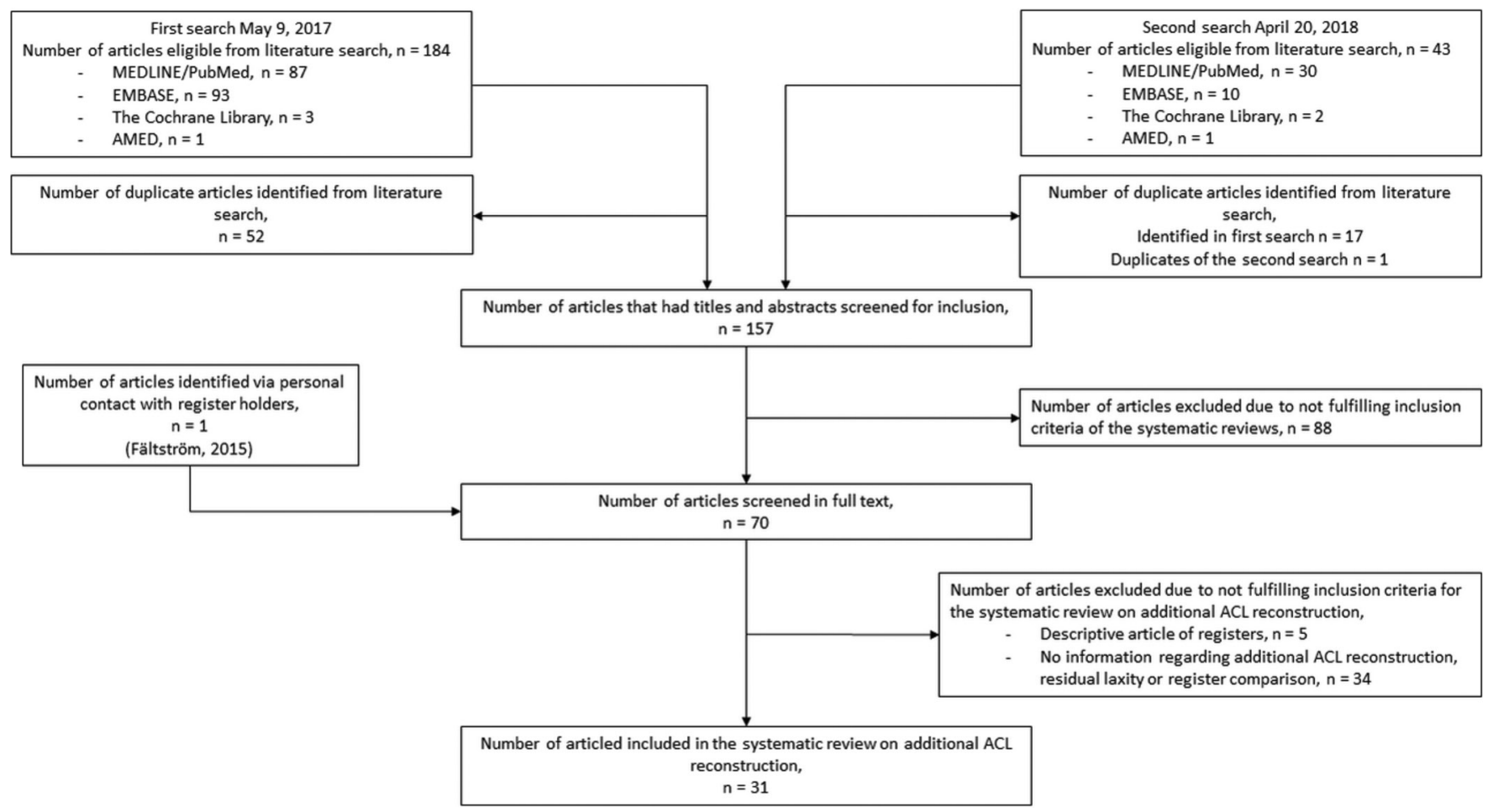

Figure 1 Study selection.

26) were the least fulfilled items (five studies fulfilled item 12 and seven studies fulfilled item 26).

\section{Relationship between patient-related factors and revision $\mathrm{ACL}$ reconstruction \\ Patient sex}

Seven studies investigated patient sex differences in revision ACL reconstruction. ${ }^{10-16}$ These studies had a quality score of $12-18$ out of 22. Andernord et $a l^{12}$ reported that the revision ACL reconstruction rate was $1.93 \%$ for women and $1.74 \%$ for men $(p=0.383)$ in the SNKLR. There was no relationship between patient sex and revision ACL reconstruction. ${ }^{10-16}$

\section{Age}

Eight studies reported that younger age increased the risk for revision ACL reconstruction. ${ }^{12-19}$ These studies had a quality score of 13-18 out of 22 . The risk of revision decreased for every subsequent 5 -year age group, starting from an age $<15$ years. ${ }^{13}$ The highest reported hazard ratio (HR) for revision ACL reconstruction (HR 5.3 [95\% CI 3.532 to 7.883]) was found when comparing the youngest age group (13-15 years) with the oldest (36-49 years). ${ }^{16}$ Patients aged $\leq 25$ years had a threefold increased risk of revision ACL reconstruction compared with patients aged $\geq 26$ years $(95 \%$ CI 2.587 to $3.934, p<0.001$ ). From an age of 30 years, the risk for revision ACL reconstruction decreased (males: risk ratio (RR) 0.31 [95\% CI 0.20 to 0.49], $\mathrm{p}<0.001$; females: RR 0.37 [95\% CI 0.22 to 0.62 ], $\mathrm{p}<0.001) .{ }^{12}$

\section{Anthropometric data}

There were conflicting results with regard to the effect of anthropometric data and the risk of revision ACL reconstruction. These studies had a quality score of 18 out of 22. In the NKLR, a body mass index (BMI) of $<25 \mathrm{~kg} / \mathrm{m}^{2}$ increased the risk for revision ACL reconstruction (HR 1.7 [95\% CI 1.1 to 2.6], $\mathrm{p}=0.012$ ). ${ }^{19}$
In the SNKLR weight, height and BMI were not associated with the risk of revision ACL reconstruction. ${ }^{12}$

Activity at injury

Football was the most commonly reported activity at time of ACL injury. ${ }^{12} 131520$ The studies investigating the association between activity at ACL injury and risk of revision had a quality score of 15-18 out of 22. In the largest cohort, football did not increase the risk of revision compared with other sports, except for alpine activities which had lower risk of revision compared with football (HR 0.81 [95\% CI 0.66 to 1.00$]$ ). ${ }^{13}$ Injury in traffic had increased risk of revision ACL reconstruction compared with football (HR 1.44 [95\% CI 1.12 to 1.87$]$ ). ${ }^{13}$ The combination of young age, female sex and injury sustained during football was associated with an increased risk of additional ACL reconstruction (accounting for both revision and contralateral ACL reconstruction) compared with the total study population and the male football players of the same age. ${ }^{20}$

\section{Patient-reported knee function}

Patient-reported knee function, measured with the KOOS, as a predictor of revision ACL reconstruction was investigated in two studies. ${ }^{1521}$ These studies had a quality score of 18 and 19 out of 22. A lower preoperative KOOS symptoms at index ACL reconstruction increased the risk of undergoing revision ACL reconstruction (HR 0.993 [95\% CI 0.989 to 0.998], $\mathrm{p}=0.007$ ). ${ }^{15} \mathrm{~A}$ KOOS quality of life (QoL) score $<44$ at 2-year follow-up from index reconstruction increased the risk of revision by 3.7 times compared with a QoL score $>44$. For every 10 -point reduction in the QoL score, the risk for revision increased by $34 \% .{ }^{21}$ The largest mean differences at 2-year follow-up between patients who went on to a revision ACL reconstruction and patients who did not were found in the subscales sport and recreation (19.5 points) and QoL (15.8 points). ${ }^{21}$ 


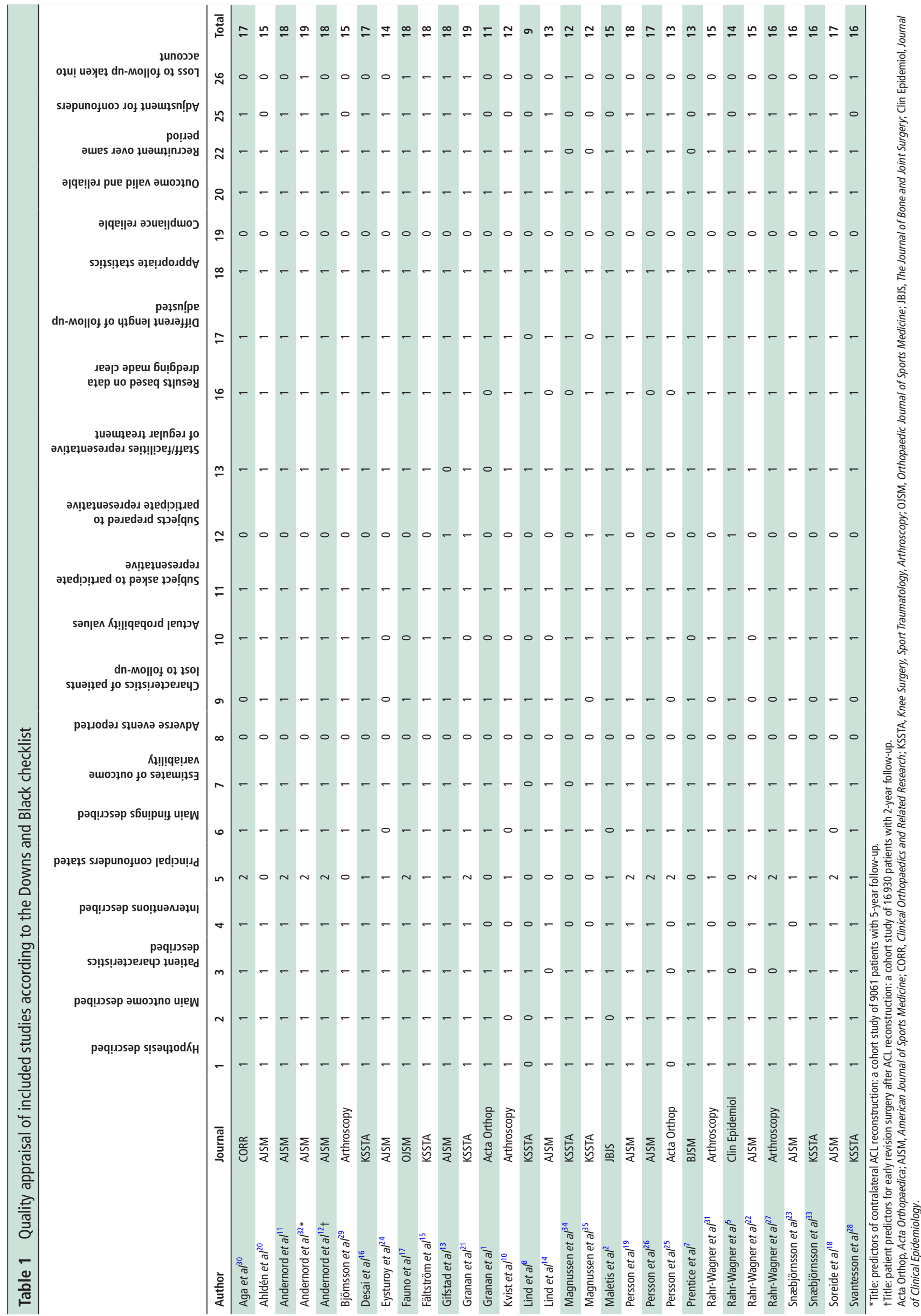


Relationship between surgical factors and revision $\mathrm{ACL}$ reconstruction

Timing of surgery

Two studies reported conflicting results on the effect of time between ACL injury and reconstruction on the risk of revision ACL reconstruction. ${ }^{1115}$ These studies had a quality score of 18 out of 22. An ACL reconstruction within less than a year from injury implied a 1.51-fold to 3.07-fold increased risk of revision compared with waiting more than a year for surgery according to the study with the largest cohort. ${ }^{15}$ In the slightly smaller study cohort, the risk of revision ACL reconstruction was not affected by the time from injury to ACL reconstruction. ${ }^{11}$

\section{Graft type}

Seven studies investigated the effect of graft choice and risk of revision. ${ }^{1011} 1315171922$ These studies had a quality score of 12-18 out of 22 . Three studies reported that the risk of revision ACL reconstruction was lower with patella tendon autografts compared with hamstring tendon autografts. ${ }^{13} 1922$ In the largest cohort, consisting of 45998 primary ACL reconstructions, the use of patella tendon autograft reduced the risk of revision by $37 \%$ compared with hamstring tendon autograft (HR 0.63 [95\% CI 0.53 to 0.74$]) .{ }^{13}$ In addition, the use of hamstring tendon autograft resulted in an almost fourfold increased risk of revision ACL reconstruction within 1 year from primary reconstruction (HR 3.82 [95\% CI 1.20 to 12.20$]$ ). ${ }^{22}$ At 5 -year follow-up, the largest difference in revision rates between hamstring tendon autograft and patella tendon autograft has been reported in patients aged $15-19$ years, 9.5\% (95\% CI 8.1 to 10.8 ) for hamstring tendon and $3.5 \%$ (95\% CI 2.1 to 4.8 ) for patella tendon autograft. ${ }^{19}$

Four studies found no difference between hamstring tendon and patella tendon autografts in terms of 2-year and 5-year risk of revision ACL reconstruction, ${ }^{5} 1011$ or 10-year risk of additional ACL reconstruction. ${ }^{15}$ No difference in the risk of revision ACL reconstruction was found depending on the use of solely semitendinosus or combined semitendinosus and gracilis grafts in hamstring tendon autograft ACL reconstructions (revision rate $2.64 \%$ vs $1.78 \%$; RR 1.48 [95\% CI 0.68 to 3.25 ], $\mathrm{p}=0.324) .{ }^{11}$

\section{Graft diameter}

There were conflicting results from two studies regarding the association between hamstring tendon autograft diameter and revision ACL reconstruction. ${ }^{11} 23$ These studies had a quality score of $16-18$ out of 22 . In one study, ${ }^{11}$ graft diameter was not associated with the incidence of revision ACL reconstruction. In a matched case-control study (with patients who went on to revision ACL reconstruction), ${ }^{23}$ the likelihood of revision ACL reconstruction was $14 \%$ lower for every $0.5 \mathrm{~mm}$ increment in graft diameter from 7.0 to $10.0 \mathrm{~mm}$ (OR 0.86 [95\% CI 0.75 to 0.99], $\mathrm{p}=0.03$ ).

\section{Graft fixation}

Five studies investigated the association between graft fixation technique and revision ACL reconstruction. ${ }^{11}{ }^{17} 24-26$ These studies had a quality score of 13-18 out of 22. Combined data from all three registers showed that transfemoral hamstring tendon autograft fixations (Rigidfix and Transfix) reduced the risk of revision by $30 \%$ compared with Endobutton. ${ }^{25}$ In the DKRR, use of cortical suspensory fixation increased the 2-year risk of revision ACL reconstruction compared with all other categories for femoral fixation (adjustable cortical suspensory fixation, intratunnel transfixation and interference screw)
(HR 1.24 [95\% CI 1.07 to 1.44], p<0.05), while intratunnel transfixation lowered the risk of revision (HR 0.83 [95\% CI 0.73 to 0.95$], \mathrm{p}<0.05){ }^{24}$ The combinations Endobutton/ Intrafix and Endobutton/Biosure PEEK for hamstring tendon autograft femoral/tibial fixation exhibited an increased risk of revision, while the lowest risk was found among combinations used for fixation of patella tendon autografts. ${ }^{24}$ Similar results have been reported also from the NKLR, ${ }^{26}$ while two studies contradict these findings by stating that femoral fixation did not influence the risk of revision. ${ }^{11}{ }^{17}$ For hamstring tendon autograft tibial fixation, the retro interference screw was associated with an increased risk of revision (HR 1.9 [95\% CI 1.3 to 2.9]) compared with an interference screw. ${ }^{25}$ The metal interference screw has also been reported to exhibit a lower risk of revision compared with all other types of tibial fixation (RR 0.32 [95\% CI 0.12 to 0.90$], p=0.031){ }^{11}$

\section{Femoral drilling technique}

Three studies assessed the influence of femoral tunnel drilling technique on the risk of undergoing revision ACL reconstruction. ${ }^{162728}$ These studies had a quality score of 16 and 17 out of 22 . Both the crude and adjusted (age, patient sex, cartilage damage, operated meniscal damage, choice of graft, prior surgery to the knee and activity leading to lesion) results of the studies were consistent in showing that there is an increased risk of revision ACL reconstruction in patients who were operated on with an anteromedial portal or transportal technique, compared with a transtibial technique to drill the femoral tunnel. The transtibial non-anatomic technique had the lowest risk of revision compared with a transportal reference technique (HR 0.694 [95\% CI 0.490 to 0.984 ], $\mathrm{p}=0.041)$. Among the transportal techniques, the less anatomical transportal technique has been reported with an increased risk of revision compared with the transportal reference anatomical technique (HR 1.310 [95\% CI 1.047 to 1.640$], \mathrm{p}=0.018) .^{16}$

\section{Concomitant injuries}

Four studies assessed the effect of concomitant injuries on the risk of undergoing revision ACL reconstruction. ${ }^{11131516}$ These studies had a quality score of 17 and 18 out of 22 . Three of the studies reported a reduced risk of revision ACL reconstruction in the presence of a cartilage injury. ${ }^{11} 1316$ The presence of a concomitant cartilage injury at time of hamstring tendon autograft ACL reconstruction decreased the 2-year risk of revision ACL reconstruction compared with the absence of a cartilage lesion ( $1.10 \%$ vs $1.86 \%$, RR 0.59 [95\% CI 0.41 to 0.84 ], $\mathrm{p}=0.004) .{ }^{11}$ One study with an up to 10 -year survival analysis contradicts these findings, and stated no association between cartilage injury at time of ACL reconstruction and subsequent revision. ${ }^{15}$ However, the authors did not present the data from the survival analysis. Among patients receiving patella tendon autograft, the concomitant cartilage injury may not reduce risk of revision ACL reconstruction. None of the studies found an association between meniscal injury and the likelihood of revision ACL reconstruction. ${ }^{11} 131516$ Concomitant injuries were more frequent in patients undergoing revision ACL reconstruction than primary reconstruction. ${ }^{20}$

\section{Single-bundle versus double-bundle $\mathrm{ACL}$ reconstruction}

Four studies investigated the relationship between single-bundle and double-bundle reconstruction, and revision ACL reconstruction. ${ }^{1128-30}$ These studies had a quality score of 16-18 out of 22. There was no difference in the risk of revision between 
double-bundle and hamstring tendon autograft single-bundle ACL reconstruction. ${ }^{30}$ However, patella tendon autograft singlebundle technique decreased the risk of revision compared with double-bundle (HR 0.62 [95\% CI 0.43 to 0.90], $\mathrm{p}=0.01$ ). ${ }^{30}$ In a separate analysis of the Swedish cohort, there was a lower risk for revision with double-bundle compared with hamstring tendon autograft single-bundle ACL reconstruction (HR $1.00 \mathrm{vs}$ 1.89 [95\% CI 1.09 to 3.29], $\mathrm{p}=0.02$ ). ${ }^{30}$ This is supported by a study from the SNKLR, reporting an increased risk of revision when using single-bundle ACL reconstruction compared with double-bundle (HR 1.98 [95\% CI 1.12 to 3.51], $\mathrm{p}=0.019$ ). ${ }^{28}$ Overall, there were no or only small differences in the risk for revision ACL reconstruction between double-bundle and singlebundle ACL reconstruction.

\section{Other factors for revision $\mathrm{ACL}$ reconstruction}

Four studies investigated the association of other factors with the risk of revision ACL reconstruction. ${ }^{12} 151831$ These studies had a quality score of $15-18$ out of 22 . One study from the $\mathrm{DKRR}^{31}$ reported that patients with simultaneous pathology involving the back had an increased risk of undergoing revision ACL reconstruction (HR 2.23 [95\% CI 1.43 to 3.48]). The risk of revision was not influenced by the administration of non-steroidal anti-inflammatory drugs (NSAIDs). However, a subgroup of patients undergoing ACL reconstruction with patella tendon autograft showed a decreased risk of revision when NSAIDs were administered (HR 0.3 [95\% CI 0.1 to 0.8 ], $\mathrm{p}=0.016$ ). ${ }^{18}$ In addition, the side of primary injury (right or left) was not associated with additional ACL reconstruction, ${ }^{15}$ and smoking or smokeless tobacco did not influence the 2-year risk of revision ACL reconstruction. ${ }^{12}$

\section{Factors associated with contralateral $\mathrm{ACL}$ reconstruction}

Four studies have investigated the risk for contralateral injury following ACL reconstruction. ${ }^{15} 193233$ These studies had a quality score of 16-19 out of 22. Age, ${ }^{15} 193233$ time to surgery (within 1 year) ${ }^{1532}$ and contralateral hamstring tendon harvest in females ${ }^{32}$ were associated with increased risk for contralateral ACL reconstruction. The highest reported RR involved age, where a 4.9-fold increased risk was found for patients aged 15-19years compared with patients over 30 years $(95 \%$ CI 3.5 to $6.9, \mathrm{p}<0.001),{ }^{19}$ followed by contralateral hamstring tendon harvest in women (RR 3.4 [95\% CI 1.4 to 7.9], $\mathrm{p}=0.006) .^{32}$ Patients who had a subsequent contralateral ACL reconstruction reported higher preoperative pain, sport/ recreation and QoL KOOS subscales of the index reconstruction compared with patients not undergoing contralateral ACL reconstruction. $^{15}$

There was conflicting evidence regarding the relationship between patient sex and contralateral ACL reconstruction (in three studies there was no relationship, ${ }^{15} 1932$ and in another study, women had higher risk of contralateral ACL reconstruction than $\mathrm{men}^{33}$ ), and the relationship between concomitant cartilage injury and contralateral ACL reconstruction (in two studies study there was no relationship, ${ }^{15} 32$ and another study reported that patients with cartilage injury had a lower risk for contralateral ACL reconstruction ${ }^{33}$ ).

There was no relationship between having a contralateral ACL reconstruction and graft type, ${ }^{1932}$ or aspects of surgical technique (tunnel drilling approach and visualisation of anatomical landmarks). ${ }^{33}$ There was also no relationship between football and other contact sports at the time of index ACL injury and having a subsequent contralateral ACL reconstruction. ${ }^{15} 32$ Although, other sports and other causes of primary injury may have a decreased risk for contralateral ACL reconstruction compared with playing football at the time of index ACL injury. ${ }^{15}$

\section{Factors for residual laxity in primary and revision ACL reconstruction}

Four studies from the DKRR reported data on residual laxity. ${ }^{14172227}$ These studies had a quality score of 13-18 out of 22. Residual rotational laxity was more common after the use of the anteromedial drilling technique compared with transtibial ${ }^{27}$ and revision ACL reconstruction cases showed less improvement in stability postoperatively compared with primary reconstructions. ${ }^{14}$ Patient age did not affect preoperative and postoperative laxity. ${ }^{17}$ The use of hamstring tendon autograft favoured regained knee joint stability compared with patella tendon autograft (OR 0.81 [95\% CI 0.68 to 0.96$]) .^{22}$

\section{The Scandinavian registers compared with data from other geographical areas}

The identified studies that compared the Scandinavian knee ligament registers with other large registers had a quality score of 11-15 out of 22 . The most recent and comprehensive study on this topic to date reported that patient characteristics were similar across the Scandinavian registers, the Luxembourg ligament register, the UK national ligament register and the US-based Kaiser Permanente Anterior Cruciate Ligament Reconstruction Register (KP ACLRR). ${ }^{7}$ However, time from injury to surgery was generally longer ( $>6$ months) in Scandinavia compared with the other cohorts, and allograft was used considerably less in the Scandinavian and other European cohorts $(\leq 1 \%)$ compared with in the US cohort (39.9\%).

Suspensory cortical fixation was the most common femoral fixation method in Scandinavia and the UK, while the Luxembourg and US registers used mostly interference fixation. The 3-year cumulative revision ACL reconstruction probability was low, ranging from 2.8 (Sweden) to 3.7 (US). The frequency of contralateral operations (any knee surgery to the contralateral knee) ranged from 1.7\% (Luxembourg) to 3.0\% (Sweden and US). ${ }^{7}$ Four studies compared data from the NKLR with the following other registers: KP ACLRR, the North American Multicenter ACL Revision Study (MARS), the Multicenter Orthopaedic Outcomes Network and the French database Société Francaise d'Arthroscopie (SFA). ${ }^{1} 23435$ Similar differences between the US registers and the NKLR were reported in these studies as in the study by Prentice et al. ${ }^{7}$

Patients in the NKLR had lower BMI compared with the KP ACLRR, ${ }^{2}$ and the US registers had a higher prevalence of meniscal and cartilage injuries at both primary and revision ACL reconstruction compared with the NKLR. ${ }^{134} 35$ Meniscal repair was more frequently performed in the US registers, while patients were more likely to undergo meniscal resection in the NKLR. ${ }^{34} 35$ Revision ACL reconstruction was most frequently performed with hamstring tendon autograft in the NKLR (56.0\%), with allograft in the MARS (49.4\%) and with patella tendon autograft in the SFA $(55.9 \%) .{ }^{35}$

\section{DISCUSSION}

This systematic review provides an overview of what is reported from the Scandinavian ACL registers with emphasis on factors associated with additional ACL reconstruction. Adolescent age was the most consistently identified risk factor associated with undergoing additional ACL reconstruction both in terms of revision and contralateral ACL reconstruction. The 
gathered findings on patients with a concomitant cartilage injury suggested a decreased risk of additional ACL reconstruction. Football players may be susceptible to an increased risk, especially in combination with young age and female sex, which may have important clinical implications for rehabilitation and return to sport (RTS). Patient sex did not affect the risk of revision ACL reconstruction, yet, female sex was significantly associated with contralateral ACL reconstruction. ${ }^{33}$

The likelihood of undergoing a revision ACL reconstruction may be additionally increased by modifiable factors for orthopaedic surgeons such as the choice of a hamstring tendon autograft over a patella tendon autograft, transportal femoral tunnel drilling, smaller graft diameter and some fixation devices. Identified factors specific for contralateral ACL reconstruction were shorter time from injury to primary ACL reconstruction, contralateral hamstring tendon harvest in women and a higher preoperative KOOS. Furthermore, there were differences between data within the Scandinavian knee ligament registers and registers in other geographical areas, especially in terms of graft choice and intra-articular findings.

Similar to the included studies in this systematic review, there are conflicting results in literature on how the use of hamstring tendon versus patella tendon autografts influences the risk of revision ACL reconstruction, ${ }^{36-39}$ and a Cochrane review found no difference in the risk of revision between the two graft choices. ${ }^{40}$ The hamstring tendon autograft is the predominant graft choice in Scandinavia, ${ }^{3713}$ and based on the gathered findings from the Scandinavian knee ligament registers, we must acknowledge the increased frequency of revision observed for hamstring tendon autografts compared with patella tendon autografts. ${ }^{13} 1922$ Nevertheless, there are several difficulties when trying to draw conclusions in this area. First, the over-representation of hamstring tendon autografts in the registers presents limitations when comparing revision rates with the considerably smaller patella tendon autograft cohort. Second, the vast majority of studies have analysed the hamstring tendon autograft as a homogenous group, although other factors influence the survival of the hamstring tendon autograft alone such as graft diameter, ${ }^{23} 4142$ fixation methods and the surgical technique. Third, the use of patella tendon autograft has gradually decreased in Scandinavia since the establishment of each register ${ }^{313}$ and a comparison with hamstring tendon autograft is therefore also a comparison of ACL reconstructions performed during different time periods. Although there were no differences in demographics between patients selected to either graft type, ${ }^{13}$ aspects such as differences in activity level and rehabilitation programmes between the graft choices were not considered in the analyses from the registers, which are key points to address in the clinical care of these patients.

The question of whether either patient sex runs a higher likelihood of revision ACL reconstruction remains unanswered in the literature. A meta-analysis concluded that women may run a slightly increased risk for revision ACL reconstruction, while on the other hand, reporting that the risk of rerupture or graft failure is similar among men and women. ${ }^{43}$ Young age, RTS and activity level play a major part in the risk of additional ACL rupture. ${ }^{44-47}$ It is therefore unfortunate that such information currently is not included in the Scandinavian knee ligament registers. However, it is possible that the finding of adolescent age as the single most established factor associated with additional ACL reconstruction is a surrogate marker for participation in knee-strenuous activity, partly explaining the increased rate of additional ACL reconstruction found in this population. There might also be selection bias, since it is possible that younger patients are more often considered for an additional ACL reconstruction.

\section{Register comparison}

The demographics of patients undergoing ACL reconstruction in different parts of the world are comparable. However, there are differences in surgical factors such as timing of surgery, graft choice, graft fixation methods and the incidence and treatment of concomitant injuries. Such surgical factors may directly influence the likelihood of additional ACL reconstruction and could therefore generate divergent findings among the registers, making it challenging to draw definite conclusions. Revision rates were similar across the registers. This might suggest that future studies determining how to decrease the incidence of additional ACL reconstruction should aim to look beyond the surgical factors and focus on the postoperative management in terms of rehabilitation and validated RTS criteria. The knowledge of how data compare between the registers can increase the understanding of contradictive findings between the registers and determine whether or not findings from one register could be translated to clinical practice in another geographical setting. As a future direction, we encourage cooperation between registers to increase the generalisability of results.

\section{Quality appraisal}

There is no validated checklist for quality appraisal of register studies and the Downs and Black checklist primarily assess the reporting quality of studies. Therefore, one must consider that a study determined to be of high reporting quality in this systematic review still could have limitations in internal validity. Adverse events (other than revision ACL reconstruction) and patient compliance to intervention (such as rehabilitation and postoperative recommendations) were not reported by any study. One reason for this is probably the limited information about adverse events in the registers, since there is no opportunity to detect graft failures or the presence of arthrofibrosis that do not proceed to additional ACL reconstruction. Patient compliance with the recommendations from surgeons and physiotherapists is a confounding factor that is always present in the Scandinavian knee ligament register studies. Finally, few studies accounted for losses to follow-up. With a few exceptions, a comparison of demographic data between study population and patients lost to follow-up was not performed. This is a problem because there may be specific subgroups of patients being included or excluded from the studies, and without an analysis on baseline characteristics of these patients there is a risk of attrition bias.

\section{Limitations}

The frequent publication from the Scandinavian knee ligament registers does induce a potential bias of using the same patient data in several studies of similar research questions. Therefore, one must beware of assigning too much importance to a single significant result among several non-significant ones. The design of register studies also implies that results should be considered as hypothesis-generating, and not proving a causal effect. It is possible that information about other knee surgeries, performed between the index ACL reconstruction and the additional ACL reconstruction, is not entered in the registers. On the other hand, the large cohort within the Scandinavian knee ligament registers still make these studies important, and are especially useful in identifying incidences, understanding practices and determining the adverse events and long-term effects of different types of exposures or interventions. ${ }^{48}$ Large registers provide 
an opportunity to perform studies with high generalisability, thereby ensuring that the results are relevant for clinicians and day-to-day practice. To appreciate these strengths and avoid possible attrition bias, it is important to continuously strive for complete data entry and compliance from both patients and surgeons in Scandinavia. Finally, this systematic review is limited by the fact that no preanalysis trial registration was registered.

\section{CONCLUSION}

Young age at index ACL reconstruction was associated with an increased risk for additional ACL reconstruction. The combination of young age, playing football at injury and female sex may elevate the risk for additional ACL reconstruction. Hamstring tendon autografts were associated with a higher risk for revision ACL reconstruction compared with patella tendon autografts, but an increased hamstring tendon graft diameter was protective. The transportal femoral drilling technique increased the risk for revision ACL reconstruction, while concomitant cartilage lesions reduced the risk of additional ACL reconstruction. There was inconclusive evidence for a relationship between additional ACL reconstruction and graft fixation, timing of surgery and singlebundle versus double-bundle technique.

\section{What are the new findings?}

- Patient sex as an independent factor does not affect the likelihood of undergoing revision $\mathrm{ACL}$ reconstruction.

- Studies from the Scandinavian knee ligament registers report different findings in regard to how surgical factors such as graft choice, tunnel drilling and fixation devices influence the risk of additional $\mathrm{ACL}$ reconstruction.

- Studies presented from the Scandinavian knee ligament registers in general have a high reporting quality but there are some differences compared with registers in other geographic areas which may limit generalisability.

\section{How might it impact on clinical practice in the future?}

- The results reflect clinical practice in Scandinavia with high generalisability and the findings should be implemented in treatment algorithms and for individualising $\mathrm{ACL}$ reconstruction.

- Continuous evaluation of outcome after $\mathrm{ACL}$ reconstruction by high completeness and compliance to the national registries should be a priority for surgeons and others practising the field.

- The results highlight subgroups of patients with potentially high-risk characteristics, which can aid providers in decision-making and helping patients set realistic outcome expectations.

Acknowledgements The authors would like to acknowledge Therese Svanberg from the library at the Sahlgrenska University hospital for help with the electronic search of the systematic review.

Contributors ES, EHS and KS have substantial contributed to the acquisition of data, analysis of data and are responsible for drafting the work and revising it critically for important intellectual content. FF, ORA and AB have done substantial contributions to the conception and design of the work, including interpretation of data. LE and JK have done large contributions in revising and designing the manuscript. All authors have given their final approval of the manuscript to be published. In addition, all authors are in agreement to be accountable for all aspects of the work in ensuring that questions related to the accuracy or integrity of any part of the work are appropriately investigated and resolved.
Funding The authors have not declared a specific grant for this research from any funding agency in the public, commercial or not-for-profit sectors.

Competing interests None declared.

Patient consent Not required.

Provenance and peer review Not commissioned; externally peer reviewed.

Data sharing statement Not applicable.

\section{REFERENCES}

1 Granan LP, Inacio MC, Maletis GB, et al. Intraoperative findings and procedures in culturally and geographically different patient and surgeon populations: an anterior cruciate ligament reconstruction registry comparison between Norway and the USA. Acta Orthop 2012;83:577-82.

2 Maletis GB, Granan LP, Inacio MC, et al. Comparison of community-based ACL reconstruction registries in the U.S. and Norway. J Bone Joint Surg Am 2011;93 Suppl 3(Suppl 3):31-6.

3 Granan LP, Forssblad M, Lind M, et al. The Scandinavian ACL registries 2004-2007: baseline epidemiology. Acta Orthop 2009:80:563-7.

4 Granan LP, Bahr R, Steindal K, et al. Development of a national cruciate ligament surgery registry: the Norwegian National Knee Ligament Registry. Am I Sports Med 2008:36:308-15.

5 Rahr-Wagner L, Thillemann TM, Lind MC, et al. Validation of 14,500 operated knees registered in the Danish Knee Ligament Reconstruction Register: registration completeness and validity of key variables. Clin Epidemiol 2013;5:219-28.

6 Engebretsen L, Forssblad M, Lind M. Why registries analysing cruciate ligament surgery are important. Br J Sports Med 2015;49:636-8.

7 Prentice HA, Lind M, Mouton C, et al. Patient demographic and surgical characteristics in anterior cruciate ligament reconstruction: a description of registries from six countries. Br J Sports Med 2018;52:716-22

8 Lind $M$, Menhert F, Pedersen AB. The first results from the Danish ACL reconstruction registry: epidemiologic and 2 year follow-up results from 5,818 knee ligament reconstructions. Knee Surg Sports Traumatol Arthrosc 2009;17:117-24.

9 Downs SH, Black N. The feasibility of creating a checklist for the assessment of the methodological quality both of randomised and non-randomised studies of health care interventions. J Epidemiol Community Health 1998;52:377-84.

10 Kvist J, Kartus J, Karlsson J, et al. Results from the Swedish national anterior cruciate ligament register. Arthroscopy 2014;30:803-10.

11 Andernord D, Björnsson H, Petzold M, et al. Surgical Predictors of Early Revision Surgery After Anterior Cruciate Ligament Reconstruction: Results From the Swedish National Knee Ligament Register on 13,102 Patients. Am I Sports Med 2014;42:1574-82.

12 Andernord $\mathrm{D}$, Desai $\mathrm{N}$, Björnsson $\mathrm{H}$, et al. Patient predictors of early revision surgery after anterior cruciate ligament reconstruction: a cohort study of 16,930 patients with 2-year follow-up. Am I Sports Med 2015;43:121-7.

13 Gifstad T, Foss OA, Engebretsen L, et al. Lower risk of revision with patellar tendon autografts compared with hamstring autografts: a registry study based on 45,998 primary ACL reconstructions in Scandinavia. Am J Sports Med 2014:42:2319-28.

14 Lind $M$, Menhert F, Pedersen AB. Incidence and outcome after revision anterior cruciate ligament reconstruction: results from the Danish registry for knee ligament reconstructions. Am J Sports Med 2012;40:1551-7.

15 Fältström A, Hägglund M, Magnusson H, et al. Predictors for additional anterio cruciate ligament reconstruction: data from the Swedish national ACL register. Knee Surg Sports Traumatol Arthrosc 2016:24:885-94.

16 Desai N, Andernord D, Sundemo D, et al. Revision surgery in anterior cruciate ligament reconstruction: a cohort study of 17,682 patients from the Swedish National Knee Ligament Register. Knee Surg Sports Traumatol Arthrosc 2017;25:1542-54.

17 Faunø P, Rahr-Wagner L, Lind M. Risk for Revision After Anterior Cruciate Ligament Reconstruction Is Higher Among Adolescents: Results From the Danish Registry of Knee Ligament Reconstruction. Orthop I Sports Med 2014;2:2325967114552405.

18 Soreide E, Granan LP, Hiorthaug GA, et al. The Effect of Limited Perioperative Nonsteroidal Anti-inflammatory Drugs on Patients Undergoing Anterior Cruciate Ligament Reconstruction. Am J Sports Med 2016:44:3111-8.

19 Persson A, Fjeldsgaard K, Gjertsen JE, et al. Increased risk of revision with hamstring tendon grafts compared with patellar tendon grafts after anterior cruciate ligament reconstruction: a study of 12,643 patients from the Norwegian Cruciate Ligament Registry, 2004-2012. Am J Sports Med 2014;42:285-91.

20 Ahldén M, Samuelsson K, Sernert N, et al. The Swedish National Anterior Cruciate Ligament Register: a report on baseline variables and outcomes of surgery for almost 18,000 patients. Am J Sports Med 2012;40:2230-5.

21 Granan LP, Baste V, Engebretsen L, et al. Associations between inadequate knee function detected by KOOS and prospective graft failure in an anterior cruciate ligament-reconstructed knee. Knee Surg Sports Traumatol Arthrosc 2015;23:1135-40

22 Rahr-Wagner L, Thillemann TM, Pedersen AB, et al. Comparison of hamstring tendon and patellar tendon grafts in anterior cruciate ligament reconstruction in a nationwide population-based cohort study: results from the danish registry of knee ligament reconstruction. Am J Sports Med 2014;42:278-84. 
23 Snaebjörnsson T, Hamrin Senorski E, Ayeni OR, et al. Graft Diameter as a Predictor for Revision Anterior Cruciate Ligament Reconstruction and KOOS and EQ-5D Values: A Cohort Study From the Swedish National Knee Ligament Register Based on 2240 Patients. Am J Sports Med 2017;45:2092-7.

24 Eysturoy NH, Nissen KA, Nielsen T, et al. The Influence of Graft Fixation Methods on Revision Rates After Primary Anterior Cruciate Ligament Reconstruction. Am I Sports Med 2018;46:524-30

25 Persson A, Gifstad T, Lind M, et al. Graft fixation influences revision risk after ACL reconstruction with hamstring tendon autografts. Acta Orthop 2018;89:204-10.

26 Persson A, Kjellsen AB, Fjeldsgaard K, et al. Registry data highlight increased revision rates for endobutton/biosure $\mathrm{HA}$ in $\mathrm{ACL}$ reconstruction with hamstring tendon autograft: a nationwide cohort study from the Norwegian Knee Ligament Registry, 2004-2013. Am J Sports Med 2015;43:2182-8.

27 Rahr-Wagner L, Thillemann TM, Pedersen AB, et al. Increased risk of revision after anteromedial compared with transtibial drilling of the femoral tunnel during primary anterior cruciate ligament reconstruction: results from the Danish Knee Ligament Reconstruction Register. Arthroscopy 2013;29:98-105.

28 Svantesson E, Sundemo D, Hamrin Senorski E, et al. Double-bundle anterior cruciate ligament reconstruction is superior to single-bundle reconstruction in terms of revision frequency: a study of 22,460 patients from the Swedish National Knee Ligament Register. Knee Surg Sports Traumatol Arthrosc 2017;25:3884-91.

29 Björnsson $\mathrm{H}$, Andernord $\mathrm{D}$, Desai $\mathrm{N}$, et al. No difference in revision rates between single- and double-bundle anterior cruciate ligament reconstruction: a comparative study of 16,791 patients from the Swedish national knee ligament register. Arthroscopy 2015;31:659-64

30 Aga C, Kartus JT, Lind M, et al. Risk of Revision Was Not Reduced by a Double-bundle ACL Reconstruction Technique: Results From the Scandinavian Registers. Clin Orthop Relat Res 2017:475:2503-12.

31 Rahr-Wagner L, Thillemann TM, Lind M, et al. Comorbidities in Patients With Anterior Cruciate Ligament Reconstruction Compared With Matched Controls Without Anterior Cruciate Ligament Injury From Danish Registries. Arthroscopy 2015;31:1741-7.

32 Andernord D, Desai N, Björnsson $\mathrm{H}$, et al. Predictors of contralateral anterior cruciate ligament reconstruction: a cohort study of 9061 patients with 5-year follow-up. Am J Sports Med 2015;43:295-302.

33 Snaebjörnsson T, Hamrin Senorski E, Sundemo D, et al. Adolescents and female patients are at increased risk for contralateral anterior cruciate ligament reconstruction: a cohort study from the Swedish National Knee Ligament Register based on 17,682 patients. Knee Surg Sports Traumatol Arthrosc 2017;25:3938-44.

34 Magnussen RA, Granan LP, Dunn WR, et al. Cross-cultural comparison of patients undergoing $\mathrm{ACL}$ reconstruction in the United States and Norway. Knee Surg Sports Traumatol Arthrosc 2010;18:98-105.
35 Magnussen RA, Trojani C, Granan LP, et al. Patient demographics and surgical characteristics in ACL revision: a comparison of French, Norwegian, and North American cohorts. Knee Surg Sports Traumatol Arthrosc 2015;23:2339-48.

36 Mohtadi N, Chan D, Barber R, et al. Reruptures, Reinjuries, and Revisions at a Minimum 2-Year Follow-up: A Randomized Clinical Trial Comparing 3 Graft Types for ACL Reconstruction. Clin I Sport Med 2016;26:96-107.

37 Xie X, Liu X, Chen Z, et al. A meta-analysis of bone-patellar tendon-bone autograft versus four-strand hamstring tendon autograft for anterior cruciate ligament reconstruction. Knee 2015;22:100-10.

38 Webster KE, Feller JA, Hartnett N, et al. Comparison of Patellar Tendon and Hamstring Tendon Anterior Cruciate Ligament Reconstruction: A 15-Year Follow-up of a Randomized Controlled Trial. Am J Sports Med 2016;44:83-90.

39 Samuelsen BT, Webster KE, Johnson NR, et al. Hamstring Autograft versus Patellar Tendon Autograft for ACL Reconstruction: Is There a Difference in Graft Failure Rate? A Meta-analysis of 47,613 Patients. Clin Orthop Relat Res 2017;475:2459-68.

40 Mohtadi NG, Chan DS, Dainty KN, et al. Patellar tendon versus hamstring tendon autograft for anterior cruciate ligament rupture in adults. Cochrane Database Syst Rev 2011:).

41 Conte EJ, Hyatt AE, Gatt CJ, et al. Hamstring autograft size can be predicted and is a potential risk factor for anterior cruciate ligament reconstruction failure. Arthroscopy 2014;30:882-90.

42 Magnussen RA, Lawrence JT, West RL, et al. Graft size and patient age are predictors of early revision after anterior cruciate ligament reconstruction with hamstring autograft. Arthroscopy 2012;28:526-31.

43 Tan SH, Lau BP, Khin LW, et al. The Importance of Patient Sex in the Outcomes of Anterior Cruciate Ligament Reconstructions: A Systematic Review and Meta-analysis. Am J Sports Med 2016:44:242-54.

44 Wiggins AJ, Grandhi RK, Schneider DK, et al. Risk of Secondary Injury in Younger Athletes After Anterior Cruciate Ligament Reconstruction: A Systematic Review and Meta-analysis. Am J Sports Med 2016;44:1861-76.

45 Paterno MV, Rauh MJ, Schmitt LC, et al. Incidence of contralateral and ipsilateral anterior cruciate ligament $(\mathrm{ACL})$ injury after primary $\mathrm{ACL}$ reconstruction and return to sport. Clin J Sport Med 2012;22:116-21.

46 Paterno MV, Rauh MJ, Schmitt LC, et al. Incidence of Second ACL Injuries 2 Years After Primary ACL Reconstruction and Return to Sport. Am I Sports Med 2014;42:1567-73.

47 Brophy RH, Schmitz L, Wright RW, et al. Return to play and future ACL injury risk after $\mathrm{ACL}$ reconstruction in soccer athletes from the Multicenter Orthopaedic Outcomes Network (MOON) group. Am I Sports Med 2012;40:2517-22.

48 Inacio MC, Paxton EW, Dillon MT. Understanding Orthopaedic Registry Studies: A Comparison with Clinical Studies. J Bone Joint Surg Am 2016;98:e3. 